T. KASHIWADA

KODAI MATH. J

3 (1980), 70-82

\title{
ON V-HARMONIC FORMS IN COMPACT LOCALLY CONFORMAL KÄHLER MANIFOLDS WITH THE PARALLEL LEE FORM
}

\author{
By TOYOKO KasHIWADA
}

Introduction. A locally conformal Kähler manifold (1.c. K-manifold) has been studied by I. Vaisman [8]. Especially when its Lee form is parallel, the manifold seems to have properties exceedingly similar to that of a Sasakian manifold. In this paper, we consider certain forms which correspond to $\mathrm{C}\left(\mathrm{C}^{*}\right)$ harmonic forms of a Sasakian manifold and with it we have some informations on the Betti number of the manifold by a decomposition of such forms. The main result is that in a $2 m$-dimensional compact $1 . \mathrm{c}$. K-manifold with the parallel Lee form, the following relation holds good between the $p$-th $(p<m)$ Betti number $b_{p}$ and the dimension $a_{p}$ of the vector space of certain $p$-forms which are defined in $\S 2$ :

$$
\begin{gathered}
b_{p}=a_{p}-a_{p-2}, \\
a_{p}=b_{p}+b_{p-2}+\cdots+b_{p-2 r}, \quad r=\left[\frac{p}{2}\right] .
\end{gathered}
$$

$\S 1$. Preliminaries. A locally conformal Kähler manifold is characterized as a Hermitian manifold $M^{2 m}(\varphi, g), 2 m=$ the dimension, such that

$$
\nabla_{k} \varphi_{j i}=-\alpha_{j} \varphi_{k i}+\alpha^{r} \varphi_{r i} g_{k J}-\alpha_{i} \varphi_{j k}+\alpha^{r} \varphi_{\jmath r} g_{k \imath} \quad\left(\varphi_{j i}=\varphi_{j}{ }^{r} g_{r i}\right)
$$

with a closed 1-form $\alpha$ which is called the Lee form, ([2], [8]). Moreover, we assume $\nabla \alpha=0,|\alpha|=1$ and $M$ is compact throughout this paper.

In this manifold, the following formulas are valid:

$$
\begin{aligned}
& \nabla_{k} \varphi_{j i}=-\beta_{j} g_{k i}+\beta_{i} g_{k \jmath}-\alpha_{j} \varphi_{k i}+\alpha_{i} \varphi_{k \jmath}, \quad \beta_{\jmath} \stackrel{d e f}{=} \alpha^{r} \varphi_{r \jmath}, \\
& J_{j i} \stackrel{d e f}{=} \nabla_{j} \beta_{i}=-\beta_{j} \alpha_{i}+\alpha_{j} \beta_{i}-\varphi_{j i} \quad\left(=-\nabla_{\imath} \beta_{j}\right), \\
& \alpha^{r} J_{r \imath}=\beta^{r} J_{r i}=0, \quad J_{\imath}^{r} J_{r}^{l}=\beta_{i} \beta^{l}+\alpha_{i} \alpha^{l}-\delta_{\imath}{ }^{l}, \\
& \nabla_{k} \nabla_{j} \beta_{i}=-\beta^{r} R_{r k j i} \\
& \quad=\beta_{j} g_{k i}-\beta_{i} g_{k \jmath}+\left(\alpha_{j} \beta_{i}-\beta_{\jmath} \alpha_{\imath}\right) \alpha_{k},
\end{aligned}
$$

R:ceived January 23, 1979 


$$
\nabla^{r} \nabla_{r} \beta_{i}=-2(m-1) \beta_{i}
$$

Furthermore, by virtue of Ricci's identity, we have

$$
\begin{gathered}
\alpha^{r} R_{r i j k}=0, \\
R_{k h \imath r} J_{\jmath}{ }^{r}-R_{k h \jmath r} J_{\imath}{ }^{r} \\
(1)=J_{k i}\left(g_{h j}-\alpha_{h} \alpha_{j}\right)-J_{k j}\left(g_{h i}-\alpha_{h} \alpha_{\imath}\right)+J_{h j}\left(g_{k \imath}-\alpha_{k} \alpha_{\imath}\right)-J_{h i}\left(g_{k j}-\alpha_{k} \alpha_{j}\right),
\end{gathered}
$$

from which

$$
\begin{gathered}
\frac{1}{2} R_{r k h \jmath} J^{r k}=-R_{h}^{r} J_{r \jmath}+(2 m-3) J_{h \jmath}, \\
R_{k}^{r} J_{r \jmath}+R_{\jmath}^{r} J_{r k}=0, \\
R_{k h \imath}{ }^{r} J_{\jmath r}-R_{k h \jmath}^{r} J_{\imath r}=-\left({R_{\imath j k}}^{r} J_{h r}-R_{\imath j h}^{r} J_{k r}\right), \\
R_{k h r s} J_{\jmath}^{r} J_{\imath}{ }^{s}=R_{j i r s} J_{k}^{r} J_{h}^{s} .
\end{gathered}
$$

The exterior product of 1 or 2 -form $\omega$ and $p$-form $u\left(=\frac{1}{p !} u_{\imath_{1} \cdots \imath p}\right.$ $\left.d x^{\imath_{1}} \wedge \cdots \wedge d x^{2 p}\right)$ is given as

$$
\begin{aligned}
& \left.(\omega \wedge u)_{i_{1} \cdots v_{p+1}}=\sum_{k=1}(-1)^{k+1} \omega_{i_{k}} u_{\imath_{1} \cdots \hat{\imath}_{k} \cdots \imath_{p+1}} \quad \text { ( } \omega: 1 \text {-form }\right) \text {, } \\
& (\omega \wedge u)_{i_{1} \cdots \imath_{p+2}}=\sum_{k<l}(-1)^{k+l+1} \omega_{i_{k^{\imath}} l} u_{\imath_{1} \cdots \hat{\imath}_{k} \cdots \hat{\imath}_{l} \cdots \imath_{p+2}} \quad \text { ( } \omega: 2 \text {-form), }
\end{aligned}
$$

where $u_{\imath_{1} \ldots \hat{\imath}_{k} \cdots \imath_{p}}$ means $i_{k}$ is omitted, and the inner product for $p$-forms $u, v$ is

$$
(u, v)=\frac{1}{p !} \int_{M} u_{\imath_{1} \cdots \imath p} v^{\imath_{1} \cdots \imath} p d \sigma
$$

In general, the star operator $*$ in a Hermitian manifold satisfies for a $p$-forms $u, v$

where

$$
\begin{array}{ll}
* * u=(-1)^{p} u, & (* u, * v)=(u, v), \\
\delta u=-* d * u, & \Delta^{*}=* \Delta,
\end{array}
$$

$$
\begin{aligned}
(d u)_{i_{0} \cdots \imath_{p}} & =\sum_{k=0}(-1)^{k} \nabla_{\imath_{k}} u_{\imath_{0} \cdots \hat{\imath}_{k} \cdots \imath_{p}}, \quad(\delta u)_{i_{2} \cdots \imath_{p}}=-\nabla^{r} u_{r \imath_{2} \cdots \imath_{p}}, \\
(\Delta u)_{i_{1} \cdots \imath_{p}} & =(\delta d u+d \delta u)_{i_{1} \cdots \imath_{p} p} \\
& =-\nabla^{r} \nabla_{r} u_{\imath_{1} \cdots \imath_{p}}+\sum_{k} R_{\imath_{k}}{ }^{r} u_{\imath_{1} \cdots{ }^{2} \cdots \imath_{p}}+\sum_{k<l} R_{\imath_{k} l_{l}}{ }^{r s} u_{\imath_{1} \cdots r^{2} \cdots s^{2} \cdots \imath_{p}}
\end{aligned}
$$

and $u_{\imath_{1} \cdots r_{n} \cdots \imath_{p}}^{k}$ means that $r$ appears at the $k$-th position.

Let operators $e(\omega), i(\omega)$ with respect to a 1 -form $\omega$ and $L, \Lambda$ be as follows 
for a $p$-form $u$ :

$$
\begin{aligned}
& e(\omega) u=\omega \wedge u, \quad i(\omega) u=* e(\omega) * u, \\
& L u=d \beta \wedge u=(e(\beta) d+d e(\beta)) u, \\
& \Lambda u=(-1)^{p} * L * u=(i(\beta) \delta+\delta i(\beta)) u .
\end{aligned}
$$

Explicitly, these are written as

$$
\begin{aligned}
& (i(\omega) u)_{i_{2} \cdots \imath}=\omega^{r} u_{r \imath_{2} \cdots \imath_{p} p}, \\
& (L u)_{i_{1} \cdots \imath_{p+2}}=2 \sum_{k<l}(-1)^{k+l+1} \nabla_{\iota_{k}} \beta_{i_{l}} u_{\imath_{1} \cdots \hat{\imath}_{k} \cdots \hat{\imath}_{l} \cdots \imath_{p+2}}, \\
& (\Lambda u)_{i_{3} \cdots \imath_{p}}=\nabla^{r} \beta^{s} u_{r s \imath_{3} \cdots \imath_{p} p} .
\end{aligned}
$$

It should be remarked that

$$
(e(\omega) u, v)=(u, i(\omega) v), \quad(L u, v)=(u, \Lambda v) .
$$

Besides under the condition $\nabla \alpha=0$, it is valid for $\omega=\alpha, \beta$,

$$
\begin{array}{ll}
L e(\omega)=e(\omega) L, & \Lambda i(\omega)=i(\omega) \Lambda, \\
L i(\omega)=i(\omega) L, & \Lambda e(\omega)=e(\omega) \Lambda .
\end{array}
$$

Since $\omega=\alpha, \beta$ are Killing, the Lie derivative

$$
\theta(\omega)=i(\omega) d+d i(\omega)
$$

satisfies the relations $([1])$ :

$$
\theta(\omega)=-(e(\omega) \delta+\delta e(\omega))
$$

and then $\theta(\omega)$ commutes with $i(\omega), e(\omega), d$ and $\delta$ for $\omega=\alpha, \beta$ respectively. In the following we often write briefly $e, i$ (resp. $e^{\prime}, i^{\prime}$ ) instead of $e(\beta), i(\beta)$ (resp. $e(\alpha), i(\alpha))$.

We notice here that

$$
\begin{aligned}
& e i+\imath e=\text { identity, } \\
& e i^{\prime}=-i^{\prime} e, \quad e^{\prime} \imath=-i e^{\prime}, \quad i i^{\prime}=-i^{\prime} i,
\end{aligned}
$$

and

$$
\begin{array}{ll}
\Delta e-e \Delta=\delta L-L \delta, & \Delta i-i \Delta=d \Lambda-\Lambda d, \\
\Delta e^{\prime}-e^{\prime} \Delta=0, & \Delta i^{\prime}-i^{\prime} \Delta=0
\end{array}
$$

because, for any Killing vector $\omega$, the following relation holds good : 


$$
(\Delta e(\omega)-e(\omega) \Delta) u=\delta(d \omega \wedge u)-d \theta(\omega) u+\theta(\omega) d u-d \omega \wedge \delta u .
$$

We remark also $\nabla_{\beta} J_{j i}=\nabla_{\alpha} J_{j i}=0$, and from which for $\omega=\alpha, \beta$

$$
i(\omega) \nabla_{\beta}=\nabla_{\beta} i(\omega), \quad i(\omega) \nabla_{\alpha}=\nabla_{\alpha} i(\omega),
$$

where we denote $\nabla_{\omega} u_{\imath_{1} \cdots \imath_{p}}=\omega^{r} \nabla_{r} u_{\imath_{1} \cdots v_{p} p}$.

In this paper the following formulas are used frequently:

LEMMA 1.1. In a l.c. K-manifold $M^{2 m}$ with the parallel Lee form, $|\alpha|=1$, the followings hold good for any p-form $u$.

（i ） $\left(\Lambda L^{k}-L^{k} \Lambda\right) u=4 k(m-p-k) L^{k-1} u+4 k\left(e^{\prime} i^{\prime}+e i\right) L^{k-1} u$.

(i ) If $i u=\Lambda u=0, \quad r \geqq 2$,

$$
\begin{aligned}
\Lambda^{r} L^{r+s}= & 4^{r}(r+s) \cdots(1+s)\left\{(m-p-s-r) \cdots(m-p-s-1) L^{s} u\right. \\
& \left.+r(m-p-s-(r-1)) \cdots(m-p-s-1) e^{\prime} i^{\prime} L^{s} u\right\} .
\end{aligned}
$$

(ii) $(\delta L-L \delta) u=2\left(d \nabla_{\beta}-\nabla_{\beta} d\right) u+4\left((m-p) e-e i^{\prime} e^{\prime}\right) u$.

(iii) $(d \Lambda-\Lambda d) u=2\left(-\delta \nabla_{\beta}+\nabla_{\beta} \delta\right) u+4\left((p-m) i-\imath e^{\prime} i^{\prime}\right) u$.

Proof. (i) and (i)' are known by the mathematical induction. (ii): Putting

$$
(\Gamma u)_{i_{0} \cdots \imath p}=\sum_{k=0}(-1)^{k} J_{\imath_{k}}{ }^{r} \nabla_{r} u_{\imath_{0} \cdots \hat{\imath}_{k} \cdots \imath_{p}},
$$

we get by straightforward computations

$$
\begin{gathered}
\frac{1}{2}(\delta L u-L \delta u)=\Gamma u+e e^{\prime} i^{\prime} u+(2 m-p-2) e u, \\
d \nabla_{\beta} u-\nabla_{\beta} d u=\Gamma u-e e^{\prime} i^{\prime} u+p e u .
\end{gathered}
$$

(iii) is known by the dual of (ii) and the property $* \nabla_{\beta} u=\nabla_{\beta} * u$. q.e.d.

$\S 2$. $V$-harmonic forms. At first we get

LEMMA 2.1. If $u$ is harmonic p-form, then

(i) $i(\alpha) u$ and $e(\alpha) u$ are harmonic,

(ii) $\nabla_{\alpha} u=0$,

(iii) ([3]) $\Lambda u=0$ (effectıve) and $i(\beta) u=0$ provided that $p<m$.

(i) and (ii) are evident if we notice that $\theta(\omega) u=0$ for a Killing vector $\omega$, a harmonic $p$-form $u$, and $\nabla_{\alpha}=\theta(\alpha)$.

Proof of (iii): In general for any $p$-form $u$, from (1.8) and Lemma 1.1, it follows that

$$
\Delta e i u=2\left(d \nabla_{\beta} i-\nabla_{\beta} d i\right) u+e\left(\Delta i+4(m-p) e i+4 e e^{\prime} i^{\prime} i\right) u
$$


and then

$$
\begin{aligned}
(\imath е u, \Delta e \imath u) & =2\left(i e u, d \nabla_{\beta} i u-\nabla_{\beta} d \imath u\right) \\
& =2\left(\text { } е u-i \delta e u, \nabla_{\beta} \imath u\right)-2\left(\imath e u, \nabla_{\beta} d \imath u\right) \\
& =-2\left(i \delta e u, \nabla_{\beta} i u\right)-2\left(\imath e u, \nabla_{\beta} d \imath u\right),
\end{aligned}
$$

where we have used $\left(\Lambda e u, \nabla_{\beta} i u\right)=\left(\Lambda u, i \nabla_{\beta} i u\right)=0$. Hence, for a form $u$ which satisfies

$$
d \imath u=\delta e u=0,
$$

the equality

$$
(\imath e u, \Delta e i u)=0
$$

holds good. We notify beforehand that this fact will be used after again in the proof of Lemma 2.5.

Now, let $u$ be harmonic. By virtue of $\theta(\beta) u=0,(2.1)$ is satisfied and then from (2.2) it follows $\operatorname{deiu}(=L i u)=0$. So, making use of Lemma 1.1, we can obtain

$$
(-L \Lambda i u, i u)=4\left((m-p) \imath u+e^{\prime} i^{\prime} \imath u, \imath u\right),
$$

which implies $\imath u=0$ under $p<m$, and then $\Lambda u=(\delta \imath+i \delta) u=0$.

q. e. d.

Definition. A form $u$ is called $V$-harmonic if it satisfies

$$
d u=0 \quad \text { and } \quad \delta u=e(\beta) \Lambda u .
$$

As a harmonic $p$-form $(p<m)$ is effective, the following is trivial:

Proposition 2.2. A p-form $(p<m)$ is harmonic of and only if it is effective V-harmonic.

Corresponding to a well known property between a harmonic form and a Killing vector, we can get

Proposition 2.3. For any V-harmonic form $u$,

holds.

$$
\theta(\beta) u=0
$$

This property follows immediately from the Lemma:

LEMMA 2.4. For any V-harmonic form $u$, $d i(\beta) u=0$ is valid.

Proof. If $u$ is $V$-harmonic, taking account of (1.8), we get

$$
\delta d i u=\Delta i u-d \delta i u=i \Delta u+d i \delta u
$$




$$
=i d \delta u+d i \delta u=\theta(\beta) e \Lambda u \text {. }
$$

Then it follows that

$$
(d \imath u, d \imath u)=(i u, \delta d \imath u)=(i u, \theta(\beta) e \Lambda u)=0
$$

because of $e \theta(\beta)=\theta(\beta) e$.

q. e. d.

Next we shall consider orthogonal property to $\beta$ of $V$-harmonic form. For it, we provide

LEMMA 2.5. For any $V$-harmonic form $u$, it is valid that

(i) $\delta e(\beta) u=0$,

(ii) i( $\beta) u$ is V-harmonıc,

(iii) $\operatorname{Li}(\beta) u=0$.

Proof. (i) follows from $\delta e u=-\theta(\beta) u-e \delta u=0$.

(ii): $\quad d \imath u=0$ is Lemma 2.4. Next, taking account of (1.6), we have

$$
\delta i u=\Lambda u-i \delta u=\Lambda u-\imath e \Lambda u=e \Lambda \imath u .
$$

(iii): By virtue of (i) and (ii), the equality (2.1) holds good, and then (2.2) as mentioned before. Hence on account of $\delta e i u=-\theta(\beta) \imath u-e \delta i u=0((\mathrm{ii}))$, we can get

$$
(\text { deiu, } \text { deiu })=-(\text { dieu, } \text { deiu })=-(\imath e u, \Delta e i u)=0,
$$

which implies Liu $=0$.

q. e. d.

THEOREM 2.6. In a compact l.c. K-manifold $M^{2 m}(\varphi, g, \alpha)$ with the parallel Lee form, a V-harmonic p-form $u(p<m)$ is orthogonal to $\beta$, i.e., $i(\beta) u=0$.

Proof. By virtue of Lemma 2.5 and Lemma 1.1, we get

$$
-L \Lambda \imath u=4\left((m-p) i+e^{\prime} i^{\prime} i\right) u,
$$

and then

$$
\begin{aligned}
(i u,-L \Lambda i u) & =-(\Lambda \imath u, \Lambda \imath u) \\
& =4(m-p)(i u, i u)+4\left(i^{\prime} \imath u, i^{\prime} i u\right) .
\end{aligned}
$$

This equality implies $i u=0$ for $m>p$. q. e. d.

Proposition 2.7. If a p-form $u(p<m)$ is $V$-harmonic, then so is $\Lambda u$. 
Proof. Let $u$ be $V$-harmonic. About the codifferential, we know obviously $\delta \Lambda u=\Lambda \delta u=e \Lambda(\Lambda u)$.

We shall prove now $d \Lambda u=0$. On account of Lemma 1.1 and (1.7), we have

from which

$$
d \Lambda u=2\left(-\delta \nabla_{\beta} u+\nabla_{\beta} \delta u\right),
$$

$$
\begin{aligned}
(d \Lambda u, & d \Lambda u)=2\left(\Lambda u, \delta \nabla_{\beta} e \Lambda u\right) \\
& =2\left(\Lambda u,-\theta(\beta) \nabla_{\beta} \Lambda u-e \delta \nabla_{\beta} \Lambda u\right) \\
& =2\left(\theta(\beta) \Lambda u, \nabla_{\beta} \Lambda u\right)-2\left(\imath \Lambda u, \delta \nabla_{\beta} \Lambda u\right) \\
& =0,
\end{aligned}
$$

where we have used $\nabla_{\beta} e=e \nabla_{\beta}$ and the properties of Lie derivative: $\theta(\beta) \Lambda=\Lambda \theta(\beta),(\theta(\beta) v, w)=-(v, \theta(\beta) w)$ for any forms $v, w$.

q. e. d.

We can also state a $V$-harmonic form with the Laplacian as follow:

Proposition 2.8. A p-form $u(p<m)$ is $V$-harmonıc if and only if $i(\beta) u=0$ and $\Delta u=L \Lambda u$.

Proof. Necessity follows from $\Delta u=d \delta u=d e \Lambda u=L \Lambda u$ (Prop. 2.7) and Theorem 2.6.

Now we prove the sufficiency. Since

$$
\begin{aligned}
(d u, d u) & +(\delta u-e \Lambda u, \delta u-e \Lambda u) \\
& =(d u, d u)+(\delta u, \delta u)-2(e \Lambda u, \delta u)+(e \Lambda u, e \Lambda u) \\
& =(u, \Delta u)-2(\Lambda u, \Lambda u-\delta i u)+(\Lambda u, \Lambda u-e \imath \Lambda u),
\end{aligned}
$$

we know, under the assumption $\imath u=0$ and $\Delta u=L \Lambda u$, the right hand side is zero. Hence $d u=0, \delta u=e \Lambda u$, which prove our Theorem.

q. e. d.

The following Proposition provids examples of $V$-harmonic forms actually.

Proposition 2.9. The $2 k$-form $L^{k} \cdot 1$ is $V$-harmonic for any $k$.

Proof. $d\left(L^{k} \cdot i\right)=0$ is trivial. So we shall prove $\delta L^{k} \cdot 1=e \Lambda L^{k} \cdot 1$ by induction.

For $k=1$, as $\delta L \cdot 1=4(m-1) \beta, e \Lambda L \cdot 1=4(m-1) \beta$, it is satisfied. Now we assume $\delta L^{k-1} \cdot 1=e \Lambda L^{k-1} \cdot 1$. Taking account of Lemma 1.1 and $\nabla_{\beta} d \beta=0$, $i^{\prime} L^{r} \cdot 1=0$, we can obtain

$$
\begin{aligned}
\delta L^{k} \cdot 1 & =\delta L \cdot L^{k-1} \cdot 1 \\
& =L \delta L^{k-1} \cdot 1+4(m-2 k+1) e L^{k-1} \cdot 1
\end{aligned}
$$




$$
\begin{aligned}
& =L e \Lambda L^{k-1} \cdot 1+4(m-2 k+1) e L^{k-1} \cdot 1 \\
& =e \Lambda L^{k} \cdot 1
\end{aligned}
$$

q. e. d.

$\S 3$. Decomposition to harmonic forms. The purpose of this section is to study the Betti number relating with $V$-harmonic forms.

At first we consider a relation between $\Delta \Lambda$ and $\Lambda \Delta$. On account of Lemma 1.1 and $\Lambda \delta=\delta \Lambda$, we have for any $p$-form $u$

$$
\begin{aligned}
\Lambda \Delta u= & \Lambda(\delta d+d \delta) u \\
= & \delta d \Lambda u-2 \delta\left(\nabla_{\beta} \delta+2(m-p) \imath-2 \imath e^{\prime} i^{\prime}\right) u \\
& +\delta d \Lambda u-2\left(-\delta \nabla_{\beta} \delta+2(p-1-m) i \delta-2 \imath e^{\prime} i^{\prime} \delta\right) u \\
= & \Delta \Lambda u-4(p-m) \Lambda u+4\left(i \delta+\delta i e^{\prime} i^{\prime}+e^{\prime} i^{\prime} i \delta\right) u .
\end{aligned}
$$

Since

$$
\begin{aligned}
-\delta e^{\prime} i i^{\prime} u & =\theta(\alpha) i i^{\prime} u+e^{\prime} \delta i i^{\prime} u \\
& =\nabla_{\alpha} i i^{\prime} u+e^{\prime} i^{\prime} \Lambda u-e^{\prime} i^{\prime} i \delta u,
\end{aligned}
$$

taking account of $\delta i^{\prime}=-i^{\prime} \delta$ and $\theta(\alpha)=\nabla_{\alpha}$, we can get finally

$$
\Lambda \Delta u-\Delta \Lambda u=4\left\{(m-p) \Lambda+i \delta+\nabla_{\alpha} i i^{\prime}+e^{\prime} i^{\prime} \Lambda\right\} u \text {. }
$$

LEMMA 3.1. For any p-form $u$, we have

$$
\begin{aligned}
(\Lambda \Delta-\Delta \Lambda) u & =4\left\{(m-p) \Lambda+e(\alpha) i(\alpha) \Lambda-\nabla_{\alpha} i(\alpha) i(\beta)+i(\beta) \delta\right\} u \\
& =4\left\{(m-p+1) \Lambda-i(\alpha) e(\alpha) \Lambda-\nabla_{\alpha} i(\alpha) i(\beta)+i(\beta) \delta\right\} u .
\end{aligned}
$$

Taking the dual of above formula and on account of $* \nabla_{\alpha}=\nabla_{\alpha} *$ we can get LEMMA 3.2. For any p-form $u$,

$$
(L \Delta-\Delta L) u=4\left\{(p-m+1) L-e(\alpha) i(\alpha) L+e(\beta) d+\nabla_{\alpha} e(\alpha) e(\beta)\right\} u
$$

holds good.

Especially if $u$ is a $V$-harmonic $p$-form $(p<m)$, Lemma 3.2 implies

$$
\begin{aligned}
\Delta L u & =L L \Lambda u-4\left((p-m+1) L u-e^{\prime} i^{\prime} L u+\nabla_{\alpha} e^{\prime} e u\right) \\
& =L\left(\Lambda L u-4(m-p-1) u-4 e^{\prime} i^{\prime} u\right)-4\left((p-m+1) L-e^{\prime} i^{\prime} L+\nabla_{\alpha} e^{\prime} e\right) u \\
& =L \Lambda(L u)-4 \nabla_{\alpha} e^{\prime} e u,
\end{aligned}
$$

which means $L u$ is also $V$-harmonic if $\nabla_{\alpha} e^{\prime} e u=0$.

From this fact, we know that the $(2 p+1)$-form $(2 p-1<m) \alpha_{\wedge} d \beta_{\wedge \ldots \wedge} d \beta$ is $V$-harmonic, because $e^{\prime} \alpha_{\wedge} d \beta_{\wedge \ldots \wedge} d \beta=0$ and $\alpha$ is $V$-harmonic. 
Proposition 3.3. If $u$ is a harmonic p-form, then $L^{k} u$ is V-harmonic, where $2+p \leqq 2 k+p \leqq m+2$.

Proof. It is sufficient to notice

$$
\nabla_{\alpha} e^{\prime} e L^{r} u=0,
$$

which follows from Lemma 2.1 (ii), $\nabla_{\alpha} e^{\prime} e=e^{\prime} e \nabla_{\alpha}$ and $\nabla_{\alpha} d \beta=0$.

q. e. d.

THEOREM 3.4. In a compact l.c. K-manifold $M^{2 m}(\varphi, g, \alpha)$ with the parallel Lee form, any $V$-harmonic $p$-form $u(p<m)$ can be represented uniquely as

$$
u=\sum_{k=0}^{r} L^{k} \phi_{p-2 k}, \quad r=\left[\frac{p}{2}\right],
$$

where $\phi_{p-2 k}$ is harmonic $(p-2 k)$-form.

Conversely, $p$-forms $(p<m)$ of the type in the right hand side are V-harmonic.

Proof. We shall prove it by the mathematical induction. At first the case $p=0$ and 1 are trivial because a $V$-harmonic form is harmonic necessarily. We assume now its validity for $(p-2)$-form. Let $u$ be a $V$-harmonic $p$-form $(p<m)$. Since $\Lambda u_{p}$ is $V$-harmonic by virtue of Proposition 2.7, there exist harmonic $(p-2-2 k)$-forms $\psi_{p-2-2 k}$ such that

$$
\Lambda u_{p}=\sum_{k} L^{k} \psi_{p-2-2 k}
$$

Now we put

$$
v_{p-2}=\sum_{k} L^{k} \phi_{p-2-2 k}
$$

where

$$
\phi_{p-2-2 k}=\frac{\phi_{p-2-2 k}}{4(k+1)(m-p+k+1)}-\frac{e^{\prime} i^{\prime} \phi_{p-2-2 k}}{4(k+1)(m-p+k+1)(m-p+2+k)} .
$$

From Lemma 2.1, $\phi_{p-2-2 k}$ are also harmonic. By virtue of Lemma 1.1 and Lemma 2.1, it follows

$$
\begin{aligned}
\Lambda L v_{p-2}= & \sum_{k} \Lambda L^{k+1}\left(\phi_{p-2-2 k}-\frac{e^{\prime} i^{\prime} \psi_{p-2-2 k}}{m-p+2+k}\right) / 4(k+1)(m-p+k+1) \\
= & \sum_{k}\left[4(k+1)(m-p+k+1) L^{k} \phi_{p-2-2 k}+4(k+1) e^{\prime} i^{\prime} L^{k} \psi_{p-2-2 k}\right. \\
& \left.-e^{\prime} i^{\prime}\left\{4(k+1)(m-p+k+1) L^{k} \psi_{p-2-2 k}+4(k+1) e^{\prime} i^{\prime} L\right\} /(m-p+2+k)\right] \\
& \times \frac{1}{4(k+1)(m-p+k+1)} \\
= & \sum_{k} L^{k} \psi_{p-2-2 k}
\end{aligned}
$$

namely, $\Lambda L v_{p-2}=\Lambda u_{p}$. Now we define a $p$-form $\phi_{p}$ as 


$$
\phi_{p}=u_{p}-L v_{p-2} .
$$

Since $L v_{p-2}=\sum_{k} L^{k+1} \phi_{p-2-2 k}$ is $V$-harmonic because of Proposition 3.3, $\phi_{p}$ is $V$-harmonic. Moreover as $\Lambda \phi_{p}=\Lambda u_{p}-\Lambda L v_{p-2}=0, \phi_{p}$ is harmonic. Then $u_{p}=\phi_{p}+L v_{p-2}=\phi_{p}+\sum L^{k+1} \phi_{p-2-2 k}$ is the desired representation.

The uniqueness comes from the following Lemma:

LEMMA 3.5. For harmonic $p$, $q$-form $\omega, \zeta(p, q<m)$, we have

$$
\left(L^{k} \omega, L^{h} \zeta\right)=0 \quad(k \neq h) .
$$

Proof. As $i \omega=\Lambda \omega=0$, making use of Lemma 1.1 and (1.6), we know for $h<k$

$$
\Lambda^{h} L^{k} \omega=\lambda L^{k-h} \omega+\mu L^{k-h} e^{\prime} i^{\prime} \omega, \quad(\lambda, \mu=\text { const. }) .
$$

Then from the property $(L \omega, \zeta)=(\omega, \Lambda \zeta)$, the Lemma is proved.

q. e. d.

From Proposition 3.3, Theorem 3.4 and Lemma 3.5, we can get

Corollary 3.6. If $u$ is a V-harmonic p-form $(p<m)$, then so is Lu. Moreover the operator $L$ is injective.

By virtue of Theorem 3.4 and Corollary 3.6, we can now obtain the desired result :

THEOREM 3.7. In a compact $2 m$-dimensional l.c. K-manifold with the parallel Lee form, we have for $p<m$,

$$
\begin{gathered}
a_{p}=b_{p}+b_{p-2}+\cdots+b_{p-2 r}, \quad r=\left[\frac{p}{2}\right] \\
b_{p}=a_{p}-a_{p-2},
\end{gathered}
$$

where $a_{p}$ is the dimension of the vector space $V_{p}$ of all $V$-harmonic p-forms and $b_{p}$ is the p-th Betti number,

$\S 4 . \quad V^{*}$-harmonic forms. In this section we shall consider a dual form of a $V$-harmonic form.

Definition. A form $u$ is called $V^{*}$-harmonic if it satisfies

$$
d u=i(\beta) L u, \quad \delta u=0 .
$$

For example, $\beta_{\wedge} d \beta_{\wedge \ldots \wedge} d \beta$ is $V^{*}$-harmonic. From the definition, we know easily

Proposition 4.1. A p-form $u$ is $V^{*}$-harmonic if and only if the $(2 m-p)$ form *u is $V$-harmonic. 
Since $\beta_{\wedge} d \beta_{\wedge \ldots \wedge} d \beta$ is $V^{*}$-harmonic $(2 p+1)$ for any $p$, by virtue of Proposition $4.1, a_{2 m-2 p-1} \geqq 1$ is valid for any $p$. Hence combining with Proposition 2.9, we can say

THEOREM 4.2. In a compact $2 m$-dimensional l.c. K-manifold with the parallel Lee form, we have $a_{k} \geqq 1$ for any $k=0,1, \cdots 2 m$.

LEMMA 4.3. For a $V^{*}$-harmonic p-form $u$, we have
(i ) $e(\beta) u=0 \quad(p>m)$,
(ii) $\theta(\beta) u=0 \quad(\forall p)$,
(iii) $\Lambda e(\beta) u=0 \quad(\forall p)$.

Proof. (i) follows from $i * u=0,(2 m-p<m)$.

(ii) follows from Proposition 2.3, i. e., $* \theta(\beta) u=\theta(\beta) * u=0$ for any $V$-harmonic $(2 m-p)$-form $* u$.

(iii) follows from Lemma 2.5 (iii), i. e., $* \Lambda e u=(-1)^{p} L \imath * u=0$ for any $V$ harmonic $(2 m-p)$-form $* u$.

Next we shall consider a decomposition of $V^{*}$-harmonic forms. For it, we provide some Lemmas.

LEMMA 4.4. For any $p(\neq m)$, we have

$$
H_{p}=V_{p} \cap V_{p}^{*},
$$

where $V_{p}^{*}$ is the vector space of $V^{*}$-harmonic $p$-forms.

Proof. From the definition, $H_{p} \supset V_{p} \cap V_{p}^{*}$ is trivial. We shall prove $H_{p} \subset V_{p} \cap V_{p}^{*}$. For $p<m$, it holds good evidently because of $i u=\Lambda u=0\left(u \in H_{p}\right)$. As for $p>m$, taking account of that $e \Lambda u=-* \imath L * u$ and $* u$ is harmonic for a harmonic form $u$, we have also $e \Lambda u=0$ and $\imath L u=0$. Hence the Lemma is proved.

q. e. d.

LEMMA 4.5. (i) $e(\beta)$ is a homomorphism of $V_{p} \cup V_{p}^{*} \rightarrow V_{p+1}^{*}$. Especially, $e(\beta)_{I_{p}}$ is injective for $p<m$.

(ii) $i(\beta)$ is a homomorphism of $V_{p} \cup V_{p}^{*} \rightarrow V_{p-1}$. Especially, $i(\beta)_{1 V_{p}^{*}}$ is surjective for $p<m+1$.

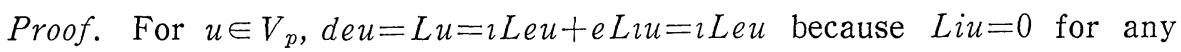
$p$ (Lemma 2.5), and $\delta e u=-\theta(\beta) u-e \delta u=0$ because $\theta(\beta) u=0$ (Prop. 2.3). Then $e u \in V_{p+1}^{*}$.

For $u \in V_{p}^{*}, d e u=L u-e d u=L u-e i L u=\imath L e u$, and as above, by virtue of Lemma $4.2, \delta e u=0$. Then $e u \in V_{p+1}^{*}$. 
Especially, for $u \in V_{p}(p<m)$, if $e u=0$, then $0=i e u=u-e \imath u=u$, namely, $e(\dot{\beta})$ is $1: 1$.

In a similar way, (ii) can be verified. Especially for non-zero $u \in V_{p-1}$, from (i), $e u \in V_{p}^{*}$, and $i e u=u-e i u=u$ for $p-1<m$.

q. e. d.

LEMMA 4.6. It is valid that for $p<m$

$$
H_{p}=i(\beta) e(\beta) V_{p}^{*}
$$

Therefore $b_{p}=0$ if any only if $e(\beta) V_{p}^{*}=\{0\}$ for $p<m$.

Proof. It is sufficient to notice that for $u \in V_{p}^{*}, V_{p} \ni \imath e u=u-e \imath u \in V_{p}^{*}$ and for $u \in H_{p}\left(\subset V_{p}^{*}\right)(p<m), u=\imath e u+e i u=\imath e u$.

q. e. d.

LEMMA 4.7. If $p<m$, we have $V_{p}^{*}=H_{p} \oplus e(\beta) V_{p-1}$.

Hence $a_{p}^{*}=b_{p}+a_{p-1}=b_{p}+\sum_{k=0}^{r} b_{p-1-2 k}\left(r=\left[\frac{p-1}{2}\right]\right)$ holds good where $a_{p}^{*}$ is the dimension of $V_{p}^{*}$.

Proof. $H_{p} \cap e V_{p-1}=\{0\}$ follows from $\imath e u=u$ for $u \in V_{p-1}(p \leqq m)$ which oppose to $i H_{p}=\{0\} \quad(p<m)$. Next, for $u \in V_{p}^{*}$, from the previous Lemmas, $u=\imath e u+e i u \in H_{p} \oplus e V_{p-1}$ is valid. Moreover from $V_{p}^{*} \supset e V_{p-1}, V_{p}^{*} \supset H_{p} \oplus e V_{p-1}$ is valid also, which completes the proof. q. e. d.

Making use of Lemma 4.7 and Theorem 3.4, we can obtain the following:

THEOREM 4.8. In a compact l.c. K-manifold $M^{2 m}(\varphi, g, \alpha)$ with the parallel Lee form, any $V^{*}$-harmonic p-form $u(p<m)$ is decomposed uniquely in the following form.

$$
u=\phi_{p}+\sum_{k=0}^{r} e(\beta) L^{k} \phi_{p-1-2 k}, \quad r=\left[\frac{p-1}{2}\right],
$$

where $\phi_{k}$ is harmonic $k$-form.

Conversely, $p$-forms $(p<m)$ of the type in the right hand side are $V^{*}$ harmonic.

Remark. Recently, Ogawa and Tachibana [6] obtain the fact that if a connected compact orientable Riemannian manifold admits a parallel vector field, then $\sum_{k=0}^{p}(-1)^{k} b_{p-k} \geqq 0$ holds good. Hence in our manifold now, as $a_{p}-a_{p-1}$ $=\sum_{k=0}^{p}(-1)^{k} b_{p-k}$ because of Theorem 3.7 , we can see the relation $a_{p} \geqq a_{p-1}$. 


\section{BIBLIOGRAPHY}

[1] S. I. Goldberg, Curvature and Homology, Academıc Press, New York, 1962.

[2] T. KASHIWADA, Some properties of locally conformal Kähler manifolds, Hokkaido Math. J., 8 (1979), 191-198.

[3] T. KAshiwAdA AND S. SATo, On harmonic forms in a compact locally conformal Kähler manifolds with the parallel Lee form, to appear.

[4] Y. OGawA, On $C$-harmonic forms in a compact Sasakian space, Tôhoku Math. J., 19 (1967), 267-296.

[5] Y. Ogawa and S. Tachibana, On Betti numbers of Riemannian manifolds with parallel vector flelds, to appear.

[36] S. TAchibana, On harmonic tensors in compact Sasakian spaces, Tôhoku Math. J., 17 (1965), 271-284.

[7] S. TAchibana, On a decomposition of $C$-harmonic forms in compact Sasakian space, Tôhoku Math. J., 19 (1967), 198-212.

[8] I. VAIsman, On locally conformal almost Kähler manifolds, Israel J. Math., 24 (1976), 338-351.

Department of Mathematics

Ochanomizu University

TOKYO, JAPAN. 